to participants. Three people responded. All three respondents said their goals were clear after the first session.

- The respondents either mostly agreed/or agreed that their symptoms were better controlled.

- Activity levels had increased.

- Ability to cope was better.

- Knowledge of helpful techniques was better and,

- Quality of life was better after attending the service for the 612 week period.

Feedback from participants includes: 'The service was friendly and positive'; 'It helped me immensely'; 'a friend had been in respiratory crisis and I was able to help her while she waited for the ambulance - that felt good'.

Conclusion Despite challenges, a virtual approach for palliative rehabilitation provided beneficial outcomes for patients who would not have been able to attend in person. This method will likely form a part of our services going forward.

\section{P-81 THERAPY AND WELLBEING WITH GRACE - TRANSFORMING OUR HOSPICE-BASED SERVICES FOLLOWING CLOSURE DUE TO COVID-19}

Kate Martin. Nottinghamshire Hospice, Nottingham, UK

10.1136/spcare-2021-Hospice.99

Background GRACE stands for Goalsetting, Reablement, Assessment, Complementary Therapy and Emotional Support. Person-centred goal setting focuses on patients' priorities for normality and functional independence (Wosahlo, 2013). Among patients' end-of-life concerns, fear of functional decline frequently eclipses fear of impending death (Cheville, 2001). Rehabilitation has been shown to reduce the impact of symptoms such as pain and anxiety and to improve functional status and quality of life (Javier \& Montagnini, 2011). The model also allows for early identification of people approaching the end-of-life and initiating discussions about preferences for end-of-life care; care planning: assessing needs and preferences, agreeing a patient and carer care plan; and coordination of care.

Aim To optimise function and wellbeing to enable people to live as well and independently as possible within the limitations of advancing illness.

Methods 2019-2020: wellbeing days piloted. May-August 2020: literature review and remodelling. Sept-December 2020: workforce planning, training in Support Needs Approach for Patients (SNAP) intervention. Jan-May 2021: recruitment, process planning.

Results The outcome measures utilised are IPOS and AKPS plus goals achieved and advance care planning. It is too early to truly evaluate the impact of our new approach.

Conclusion GRACE is a person-centred approach with a focus on reablement and wellbeing. It allows for earlier identification and support of those in their last year of life.

\section{P-82 INCREASING PALLIATIVE REHABILITATION'S REACH THROUGH TECHNOLOGY: ONLINE RESOURCES AND VIRTUAL THERAPY}

Mary Banks. Mountbatten Hospice Group, Isle of Wight, UK
Introduction Traditional hospice delivery of rehabilitation to outpatients and day service attendees involved face-to-face appointments and group sessions. For our organisation, like many others, the COVID-19 pandemic halted this, and became a catalyst for change. It was important to identify and achieve alternative methods of delivery to ensure the valuable education, support and interventions for our patients and families could continue.

Method A project team, involving a range of roles including Communications, Rehabilitation, Quality and IT was established to create and deliver a host of educational online resources accessible on the hospice website with written information, illustrations and recorded videos. A virtual timetable of sessions including Living well with Breathlessness, Exercise circuits and Adapted Yoga was created for people to access from home. Alongside this project, in order to support more people to access these developments and address any potential inequalities, a team of volunteers confident with technology were recruited to support individuals in improving IT skills, accessing online resources and joining online sessions. This even included providing a laptop on loan if needed.

Outcomes With information, advice and recorded demonstrations now readily available on the website the hospice has provided significantly greater opportunity for people to be better informed about self-management of their symptoms and condition, and subsequently has therefore furthered its reach and improved understanding of the role of palliative rehabilitation. The alternative method of accessing sessions and groups has created greater choice for individuals, and options according to their preference as to whether to travel and attend a face-to-face appointment, or access the session from their own home, or even workplace. Some of the historical and familiar barriers to accessing rehabilitation such as transport, work commitments, family commitments and the weather have been overcome as a result.

Conclusion The project will be further evaluated via website analytics, feedback questionnaires and group attendance statistics.

\section{P-83 ACCEPTABILITY OF A TELE-REHABILITATION INTERVENTION FOR FATIGUE AND BREATHLESSNESS IN PALLIATIVE CARE}

Alison Christian, Lonan A Challis, Jo Beard, Alison Snelling, Sarah M McGhee, Anne Mills. Hospice Isle of Man, Douglas, Isle of Man

10.1136/spcare-2021-Hospice. 101

Background In response to the COVID-19 pandemic, the hospice moved to digital approaches. Whilst tele-rehabilitation has shown benefits for various chronic health conditions (Bhatt, Patel, Anderson, et al., 2019; Zanaboni, Hoaas, Lien, et al., 2017; Hwang, Bruning, Morris, et al., 2017), there is a gap in the literature on telehealth interventions for palliative rehabilitation.

Aim To evaluate digital delivery of a palliative rehabilitation programme and obtain perceptions of users and staff.

Methods All members of the Fatigue and Breathlessness (FAB) follow-on group $(n=19)$ were invited to complete a questionnaire on the experience of transitioning to Zoom sessions. Descriptive statistics were produced using the statistical 
software package, Stata (Version 15; StataCorp, 2017). Qualitative data were analysed using an inductive thematic analysis framework (Braun \& Clarke, 2006). Three members of the rehabilitation team were interviewed about encountered benefits and challenges.

Results Thirteen members completed the questionnaire (68\%) and all were positive about the transformed sessions. Eight respondents (62\%) felt that the Zoom sessions were 'no different' or 'better' than in-person sessions. No adverse events were reported. Themes from open-ended comments included patient-level effects such as maintained exercise and social contact when in isolation and removed travel requirements. At the service level, there was improved access but technological challenges. Most respondents (9, 69\%) suggested keeping the option of Zoom for flexibility and $46 \%$ (6) wanted both staff-led and self-led elements.

The rehabilitation team felt their rapid response and team working enabled efficient transition to Zoom. This included risk assessments, particularly for those living alone. With help, users quickly learned and the virtual delivery provided opportunities to try new activities. At times, staff found the 'silent audience' challenging. The rehabilitation team felt the approach may only work with groups with existing rapport.

Conclusions The hospice rehabilitation team now provide concurrent sessions at home via Zoom and in the hospice. These access options provide choice, appear to be acceptable and offer flexibility around changing condition status and personal factors.

\section{P-84 VIRTUAL WELLBEING AT THE KIRKWOOD- 'YOU'RE ON MUTE...'}

Mary Tyrrell Place, Joanne Keeling. The Kirkwood, Huddersfield, UK

10.1136/spcare-2021-Hospice.102

In March 2020, due to the increasing numbers of people affected by the coronavirus pandemic in Kirklees, a number of The Kirkwood's services were suspended, including those which involve public gatherings of patients, carers, family members and volunteers - this included all of the wellbeing and self-management information sessions for people affected by life-limiting conditions. Following trials in virtual delivery of small pockets of this programme in the latter part of 2020, a virtual wellbeing programme was launched. It was identified that there was a lot of duplication within the different self-management modules and the decision was made to minimise this and bring the different groups of patients and carers together to cover the more generic topics such as breathlessness management, living with fatigue and sleep promotion. This resulted in decreased burden on our community colleagues who were under immense pressure due to COVID19.

Sessions took place from Tuesday to Friday over Zoom and covered different aspects of self-management and general wellbeing. These included information sessions around living with fatigue, managing breathlessness and sleep promotion. More generic wellbeing sessions were also provided which covered self-care, social interaction and music therapy. Partnerships with pre-COVID-19 partners such as Huddersfield Town Foundation were re-established and joint sessions (e.g. Sporting
Memories ) were delivered virtually. New partnerships were developed (Nordoff -Robbins) and initiatives such as an online choir were developed and thrived.

Patient feedback was collected in real time and retrospectively after a 12-week period. Real time feedback was overwhelmingly positive and constructive with a consistent emphasis on their helpfulness, relevance, inclusiveness and enjoyability. The main themes of the retrospective feedback were that the sessions were easy to access, useful, informative and social.

When asked if we could have done anything differently, the only request from some was for face-to-face sessions when restrictions allowed.

\section{P-85 HOSPICE VIRTUAL WELLBEING SERVICE: SUPPORTING PATIENTS AND CARERS ONLINE THROUGH THE PANDEMIC}

Sophia Monastirioti, Fiona McMinn, Heather Phillips, Nicola Brennan, Laura Yalley-Ogunro. Princess Alice Hospice, Esher, UK

\subsection{6/spcare-2021-Hospice. 103}

Background Wellbeing centres across the UK have been closed to face-to-face attendances by terminally ill patients and their carers since the start of the COVID-19 pandemic in March 2020 (Swann, Easton, McGuinness, et al., 2021). Day hospices have had to be flexible (Dunleavy, Preston, Bajwah, et al., 2021; Etkind, Bone, Lovell, et al., 2020; Stevens, Martin, White, 2011) and think creatively (Roberts, 2021) to maintain support for our patients. We developed a virtual programme to continue our high level of support for our patients and carers.

Aims We aimed to offer a wide variety of virtual sessions to our patients and their loved ones to help with symptom control and maintain social and psychological support (Stevens, Martin, White, 2011).

Methods We provided virtual group sessions such as yoga, exercise classes, guided relaxation, art, Q\&A sessions with our doctors, discussion support groups, carers' groups, management of breathlessness and pain, anxiety and fatigue management, in addition to one-to-one nursing assessments. Our complementary therapist offered aromatherapy education and our physiotherapists supported patients to maintain mobility and physical function. Two online surveys captured service users' experience of the virtual programme.

Results Our first virtual session was held on 1 April 2020 with three participants attending. This grew to 18 scheduled sessions per week supporting an average of 23 patients a day. Up to April 2021, we had 4,525 attendances and 371 external referrals. Our survey showed that attendees felt the online sessions improved mood (91.2\%) and helped to improve symptom management (84.2\%).

Conclusions Our patients and carers showed keen enthusiasm for the virtual wellbeing sessions. We plan to continue to offer this support going forward alongside a return to face-toface sessions when possible with the advantage of extending the reach of our service to patients and carers who may not be able to attend in person. Maintaining a link with the hospice during the pandemic and social interaction for our patients who have been shielding at home has been appreciated. 